\title{
Brolucizumab and immunogenicity
}

\author{
Ashish Sharma ${ }^{1}$ Nilesh Kumar ${ }^{1}{ }^{1} \cdot$ Nikulaa Parachuri $^{1} \cdot$ Rohini Sharma $^{2} \cdot$ Francesco Bandello $^{3}$. \\ Baruch D. Kuppermann ${ }^{4}$. Anat Loewenstein ${ }^{5}$
}

Received: 27 February 2020 / Revised: 2 March 2020 / Accepted: 10 March 2020 / Published online: 6 April 2020

(c) The Royal College of Ophthalmologists 2020

Brolucizumab (Beovu, Novartis, Basel, Switzerland) was recently approved by the US FDA in October 2019 for the treatment of neovascular age-related macular degeneration (n-AMD). It is a single-chain antibody fragment (scFv), the smallest functional subunit of an antibody molecule, approved for the first time across all fields of medicine $[1,2]$.

Brolucizumab was approved on the basis of the pivotal phase 3 clinical trial results of the HAWK and HARRIER studies. Approximately $90 \%$ of the uveitis and iritis cases were mild to moderate, which were treated with a course of topical corticosteroids/antibiotics and most resolved with no sequelae [3]. Two of the 8 cases of uveitis were reported as a serious adverse event (SAE) in the $6 \mathrm{mg}$ brolucizumab group, compared to 0 cases in the aflibercept group of HAWK trial, HARRIER had three incidences of uveitis, all reported as SAE. No case of reported iritis was categorized as SAE. One case of retinal artery thrombosis was noted in each of the trials (HAWK and HARRIER) with $6 \mathrm{mg}$ of brolucizumab [3].

Recently, the American Society of Retina Specialists (ASRS) released an alert reporting that apart from mild to moderate intraocular inflammation, there have been 14 cases of vasculitis. Of which, 11 were designated as

$\triangle$ Ashish Sharma

drashish79@hotmail.com

1 Lotus Eye Hospital and Institute, Avinashi Road, Coimbatore, TN, India

2 Department of Healthcare, The TIPS Global Institute, Coimbatore, TN, India

3 University Vita-Salute, Scientific Institute San Raffaele, Milano, Italy

4 Gavin Herbert Eye Institute, University of California, Irvine, CA, USA

5 Division of Ophthalmology, Tel Aviv Sourasky Medical Center and Sackler Faculty of Medicine, Tel Aviv University, Tel Aviv, Israel occlusive retinal vasculitis. According to the manufacturer (Novartis), 46,000 injections have been administered to date in the USA [4].

It is important to understand this phenomenon in the light of the structure of these monoclonal antibodies used for retinal diseases and the historical data regarding inflammation caused by these molecules [Ranibizumab (Lucentis; Genentech, South San Francisco, CA) (Razumab; Intas Pharmaceutical Ltd, Ahmedabad, India) Bevacizumab (Avastin; Genentech, South San Francisco, CA), or Aflibercept (Eylea; Regeneron, Tarrytown, NY)].

The basic molecular structure varies with each antiVEGF aimed for intravitreal therapy [1]. Bevacizumab is a full-size humanized anti-VEGF monoclonal antibody, while ranibizumab is a humanized Fab fragment that neutralizes soluble splice variants and proteolytic fragments of VEGFA. Aflibercept (VEGF Trap) is a chimeric molecule carrying soluble receptors VEGFR1 and VEGFR2 linked to an Fc fragment of human IgG1, which essentially binds to VEGF and prevents its action. Brolucizumab is a $28 \mathrm{kDa}$ humanized scFv molecule that binds to all the isoforms of VEGF$\mathrm{A}$ and renders them ineffective. Structural modifications were achieved to improve the efficacy and duration of action of these drugs. Brolucizumab has shown significantly better fluid control across all the layers with a longer duration of action in the HAWK and HARRIER trials [3]. It was hypothesized that it could be due to the smaller structure of the molecule which led to a better penetration in the retinal layers [3]. Monoclonal antibodies are complex protein molecules and may cause immunogenicity [5]. However, it is difficult to assess whether the structure of these molecules has a relationship with immunogenicity.

Ocular inflammation has been reported with all the antiVEGF monoclonal antibodies in use for intravitreal therapy prior to the approval of brolucizumab [6]. Kiss et al. reported that the rates of endophthalmitis following aflibercept, bevacizumab and ranibizumab injections were $0.100 \%$ (136/135,973), 0.056\% (268/481,572), and 0.047\% (94/201,013), respectively [6]. Souied et al. analyzed a 
retrospective claims database which included 432,794 injection claims (ranibizumab $n=253,647$, aflibercept $n=$ 179,147). They have reported that significantly higher, unique, and severe ocular inflammatory events occurred more in patients receiving aflibercept than ranibizumab (1.06/1000 injections of aflibercept, $95 \%$ confidence interval [CI], $0.91-1.21$ vs. $0.64 / 1000$ injections of ranibizumab, 95\% CI $0.54-0.74 ; p<0.0001$ ) [7].

In the recent ASRS alert for brolucizumab, the percentage of vasculitis is $0.03 \%$ (14/46000) (excluding mild and moderate inflammation for which the data is not available to the authors) [4]. This is in line with the inflammation data of other anti-VEGFs [6, 7]. Therefore ASRS alert should not be seen in isolation and create a nocebo effect [8]. Biosimilar of ranibizumab (Razumab) faced a similar situation when it was launched in 2015 in India [9]. A total of 10\% of patients who received products from the first three batches of the biosimilar experienced inflammation. Intas introduced an additional filtration step to its manufacturing process and added fresh "ultrapure Polysorbate 20". [10] Furthermore, endotoxin limits were also re-defined and corrected [11]. After addressing these manufacturing issues, no cases of inflammation have been reported further with the drug.

However, there is a minor difference in the inflammation profile with brolucizumab compared to inflammation reported with anti-VEGFs in the past [5-7]. Cases with occlusive vasculitis are reported with brolucizumab in addition to mild and moderate intraocular inflammation [5]. It is difficult to explain the vasculitis without knowing the details about the reported cases such as whether the vasculitis was seen in cases with extensive uveal inflammation or if it was an isolated phenomenon. A 30-year surveillance analysis of adverse events following systemic mAb therapy noted 79 cases of biopsy-proven vasculitis, 69 of which were administered for auto-immune conditions [12]. Type III hypersensitivity reactions (HSR) to the mAbs including anti-VEGF agents used for oncological indications have been reported to cause vasculitis [13]. Aggregates of $\mathrm{IgG} / \mathrm{IgM}$ complex deposit in the local tissue upon drug administration, termed as Arthus reaction, the type III HSR noted with the injection of systemic mAb [14]. An Occurrence of similar reactions may be plausible in the cases of vasculitis related to brolucizumab, though it can't be proven conclusively at this point in time.

These immunogenic reactions to the drug raise the question about their occurrence in an immune-privileged organ like the eye where the adaptive immune reaction is not in play under normal circumstances. We have previously described the possible mechanisms of immunogenicity responsible for ocular inflammation caused by the biologics used in the management of retinal diseases [15]. An extensive review of the case records of the patients developing vasculitis, with a special emphasis on the integrity of the ocular-blood barrier (association with uveal inflammation or diabetes) may provide further insight into the mechanism.

To conclude, ASRS alert about brolucizumab should be used as a safety measure to select the patients meticulously at this stage such as avoiding the use of the drug in the patients who have preexisting inflammation. Furthermore, careful counseling and follow-up of patients who are receiving the injection is needed. This alert could also help the manufacturer to find out the cause and address it.

\section{Compliance with ethical standards}

Conflict of interest AS: consultant: for Novartis, Allergan, Bayer and Intas. FB: consultant: Allergan, Bayer, Boehringer-Ingelheim, Fidia Sooft, Hofmann La Roche, Novartis, NTC Pharma, Sifi, Thrombogenics, Zeiss. BDK: clinical research: Alcon, Alimera, Allegro, Allergan, Apellis, Clearside, Genentech, GSK, Ionis, jCyte, Novartis, Regeneron, ThromboGenics; consultant: Alimera, Allegro, Allergan, Cell Care, Dose, Eyedaptic, Galimedix, Genentech, Glaukos, Interface Biologics, jCyte, Novartis, Ophthotech, Regeneron, Revana, Theravance Biopharma. AL reports other from Allergan, other from Novartis, other from Roche, other from Notal Vision, other from Forsightslabs, other from Beyeonics, other from Bayer Health Care. NK, NP, and RS: none

Publisher's note Springer Nature remains neutral with regard to jurisdictional claims in published maps and institutional affiliations.

\section{References}

1. Sharma A, Kumar N, Kuppermann BD, Bandello F. Brolucizimab-leading an era of structural revolution for long-term VEGF suppression. Eye (Lond). 2020;34:611-3.

2. Antonio Ligi. Novartis receives FDA approval for $B e o v u^{\circledR}$, offering wet AMD patients vision gains and greater fluid reductions vs aflibercept. https://www.novartis.com/news/media-relea ses/novartis-receives-fda-approval-beovu-offering-wet-amd-pa tients-vision-gains-and-greater-fluid-reductionsvs-aflibercept. Accessed Mar 2020.

3. Dugel PU, Koh A, Ogura Y, Jaffe GJ, Schmidt-Erfurth U, Brown DM, et al. HAWK and HARRIER: phase 3, multicenter, randomized, double-masked trials of brolucizumab for neovascular agerelated macular degeneration. Ophthalmology. 2020;127:72-84.

4. Clinical Updates: Beovu Update for ASRS Members. 2020. https://www.asrs.org/clinical/clinical-updates/960/Beovu-Updatefor-ASRS-Members

5. Kuriakose A, Chirmule N, Nair P. Immunogenicity of biotherapeutics: causes and association with posttranslational modifications. J Immunol Res. 2016;2016:1298473.

6. Kiss S, Dugel PU, Khanani AM, Broder MS, Chang E, Sun GH, et al. Endophthalmitis rates among patients receiving intravitreal anti-VEGF injections: a USA claims analysis. Clin Ophthalmol. 2018; 12:1625-35.

7. Souied EH, Dugel PU, Ferreira A, Hashmonay R, Lu J, Kelly SP. Severe ocular inflammation following ranibizumab or aflibercept injections for age-related macular degeneration: a retrospective claims database analysis. Ophthalmic Epidemiol. 2016;23:71-9.

8. Sharma A, Kumar N, Bandello F, Loewenstein A, Kuppermann BD. Need of education on biosimilars amongst ophthalmologists: 
combating the nocebo effect. Eye. 2019. https://doi.org/10.1038/ s41433-019-0722-6.

9. EXCLUSIVE: complaints prompt Intas to recall batch of biosimilar Razumab in India. 2020. https://www.moneycontrol.com/ news/business/companies/exclusive-complaints-prompt-intas-torecall-batch-of-biosimilar-razumab-in-india-2251273.html.

10. Laird Harrison. New Batches of ranibizumab biosimilar safe, effective. 2020. https://www.medscape.com/viewarticle/867481. Accessed Mar 2020.

11. Sharma A, Kumar N, Kuppermann BD, Bandello F, Loewenstein A. Ophthalmic biosimilars and biologics-role of endotoxins. Eye (Lond). 2020;34:614-5.

12. Lioger B, Hennekinne F, Agier MS, Jonville-Bera AP, Maillot F. Incidence and characteristics of vasculitis associated with monoclonal antibodies and peptide fusion proteins: a survey from the
French National Pharmacovigilance Database. Arthritis Rheumatol. 2016;68 suppl 10. https://acrabstracts.org/abstract/incidence-a nd-characteristics-of-vasculitis-associated-with-monoclonal-a ntibodies-and-peptide-fusion-proteins-a-survey-from-the-frenchnational-pharmacovigilance-database/. Accessed 27 Feb 2020.

13. Baldo B. Adverse events to monoclonal antibodies used for cancer therapy: focus on hypersensitivity responses. OncoImmunology 2013;2:e26333.

14. Isabwe GAC, Garcia Neuer M, de las Vecillas Sanchez L, Lynch D-M, Marquis K, Castells M. Hypersensitivity reactions to therapeutic monoclonal antibodies: phenotypes and endotypes. J Allergy Clin Immunol. 2018;142:159-170.e2.

15. Sharma A, Kumar N, Kuppermann BD, Bandello F, Loewenstein A. Biotherapeutics and immunogenicity: ophthalmic perspective. Eye. 2019;33:1359-61. 